\title{
Evidence for Spatial Modules Mediated by Temporal Synchronization of Carbachol-Induced Gamma Rhythm in Medial Entorhinal Cortex
}

\author{
Clayton T. Dickson, Gerardo Biella, and Marco de Curtis \\ Department of Experimental Neurophysiology, Istituto Nazionale Neurologico "Carlo Besta", Milan 20133, Italy
}

\begin{abstract}
Fast $(\gamma)$ oscillations in the cortex underlie the rapid temporal coordination of large-scale neuronal assemblies in the processing of sensory stimuli. Cortical $\gamma$ rhythm is modulated in vivo by cholinergic innervation from the basal forebrain and can be generated in vitro after exogenous cholinergic stimulation. Using the isolated guinea pig brain, an in vitro preparation that allows for the study of an intact cerebrum, we studied the spatial features of $\gamma$ activity evoked by the cholinomimetic carbachol (CCh) in the medial entorhinal cortex (mEC). $\gamma$ activity induced by either arterial perfusion or intraparenchymal application of CCh showed a phase reversal across mEC layer II and was reduced or abolished in a spatially localized region by focal infusions of atropine, bicuculline, and CNQX. In addition, a spatially restricted zone of $\gamma$ activity could be induced by passive diffusion of CCh from a recording pipette. Finally, $\gamma$ oscillations recorded at multiple sites
\end{abstract}

across the surface of the mEC using array electrodes during arterial perfusion of CCh demonstrated a decline in synchronization (coherence) as the interelectrode distance increased. This effect was independent of the signal amplitude and was specific for $\gamma$ as opposed to theta-like activity induced by CCh in the same experiments.

These results suggest that CCh-induced $\gamma$ oscillations in the $\mathrm{mEC}$ are mediated through direct muscarinic excitation of a highly localized reciprocal inhibitory-excitatory network located in superficial layers. We propose that functional cortical modules of highly synchronous $\gamma$ oscillations may organize incoming (cortical) and outgoing (hippocampal) information in the mEC.

Key words: $\gamma$ rhythm; $40 \mathrm{~Hz}$ oscillation; synchrony; muscarinic; entorhinal cortex; perforant path; limbic system; inhibitory interneuron; GABA; integration; binding; memory
Rhythmic oscillatory activity, through its ability to temporally synchronize the activity of large subsets of cortical neurons, has been thought to underlie both fundamental (Schurmann et al., 1997) and higher brain functions such as perception (Eckhorn et al., 1988; Gray et al., 1989), arousal or attention (Tiitinen et al., 1993; Maloney et al., 1996), sensorimotor integration (Murthy and Fetz, 1992), and even cognition or consciousness (Llinás and Ribary, 1993). Fast activity in the $\gamma$ range $(25-80 \mathrm{~Hz}$ ) has been observed throughout the cortical mantle, including limbic cortices, where it has been suggested to play a role in memory processes (Bragin et al., 1995; Lisman and Idiart, 1995; Chrobak and Buzsaki, 1998a). A nodal portion of the limbic area is the entorhinal cortex (EC), which sits at the interface between the neocortex and the hippocampus (Van Hoesen, 1982; Witter et al., 1989) and, as such, is an integral part of the temporal lobe memory system (Squire and Zola-Morgan, 1991; Zola-Morgan and Squire, 1993). A proportion of superficially located EC neurons, the cells of origin of the perforant path input to the hippocampal formation, are endowed with endogenous oscillatory properties (Alonso and Llinás, 1989) and show rhythmic and synchronized activity in vivo at both slow (theta) and fast $(\gamma)$ frequencies (Mitchell and Ranck, 1980; Alonso and García-Austt, 1987; Dickson et al., 1995; Chrobak and Buzsaki, 1998a). These oscillatory rhythms are presumably important for information processing and memory formation and retrieval (Winson, 1978; Buzsáki, 1989; Ahissar et al., 1992; Lisman and Idiart, 1995; Shulz et al., 2000). As in the neocortex (Steriade et al., 1991; Metherate et al., 1992; Munk et al., 1996; Cape and Jones, 1997; Herculano-Houzel et al., 1999), the expression and modulation of these rhythms are under the control of cholinergic inputs from the

\footnotetext{
Received June 22, 2000; revised July 28, 2000; accepted Aug. 9, 2000.

This study was supported by the Human Frontier Science Program (HFSP) Grant RG 19/96. C. Dickson was the recipient of a short term HFSP fellowship SF 9/99. G. Biella was partially sponsored by the European Community Grant VSAMUEL (IST-99-1-1-A).

Correspondence should be addressed to Marco de Curtis, Dipartimento di Neurofisiologia Sperimentale, Istituto Nazionale Neurologico "Carlo Besta", via Celoria, 11, 20133 Milan, Italy. E-mail: decurtis@istituto-besta.it.

Copyright (C) 2000 Society for Neuroscience $0270-6474 / 00 / 207846-09 \$ 15.00 / 0$
}

basal forebrain (Dickson et al., 1994; Jeffery et al., 1995; Ma and Leung, 1999), which profusely innervates the EC (Alonso and Köhler, 1984). In vitro studies have shown that cholinergic activation exerts profound modulatory effects on the membrane properties of superficial EC neurons, which allow for the expression of oscillatory behavior (Dickson and Alonso, 1997; Klink and Alonso, 1997b).

$\gamma$ oscillatory activity has been described in in vitro slice preparations of hippocampus (Fisahn et al., 1998; Fellous and Sejnowski, 2000) and somatosensory cortex (Buhl et al., 1998) treated with the cholinergic agonist carbachol (CCh). Recently, we have demonstrated that $\mathrm{CCh}$ perfusion elicits $\gamma$ activity in the medial EC (mEC), but not lateral EC (IEC) of the isolated guinea pig brain preparation (van der Linden et al., 1999). In this preparation the planar horizontal connections in cortical structures such as the EC are intact and thus allow for a controlled study of large-scale networks in the generation of synchronized oscillatory activity. The primary objective of the present study was to examine the circuit mechanisms and the spatial distribution of CCh-induced $\gamma$ oscillations within the mEC. Through a series of localized pharmacological and multisite electrophysiological experiments we show that these oscillations are dependent on the local muscarinic synchronization of spatially restricted pools of reciprocally connected superficial inhibitory and excitatory neurons. A physiological role for these oscillations may be to spatially and temporally organize into functional modules the output of mEC projection cells to their targets in the hippocampus.

Some of these results have previously appeared in abstract form (de Curtis et al., 1999).

\section{MATERIALS AND METHODS}

The isolated whole brain preparation has been previously documented in detail (de Curtis et al., 1991, 1998; Muhlethaler et al., 1993) and will only briefly be described here. After barbiturate anesthesia (thiopentothal sodium, $20 \mathrm{mg} / \mathrm{kg}$ ), young adult guinea pigs $(150-300 \mathrm{gm})$ were perfused through the heart with a cold $\left(4-10^{\circ} \mathrm{C}\right)$ carbogenated $\left(95 \% \mathrm{O}_{2}\right.$ and $5 \%$ $\mathrm{CO}_{2}$ ) solution composed of (in mM): $126 \mathrm{NaCl}, 3 \mathrm{KCl}, 1.2 \mathrm{KH}_{2} \mathrm{PO}_{4}, 1.3$ $\mathrm{MgSO}_{4}, 2.4 \mathrm{CaCl}_{2}, 26 \mathrm{NaHCO}_{3}, 15$ glucose, 2.1 HEPES, 0.4 thiourea, 0.5 ascorbic acid, and $3 \%$ dextran (molecular weight 70,000$)$. After rapid and 
A
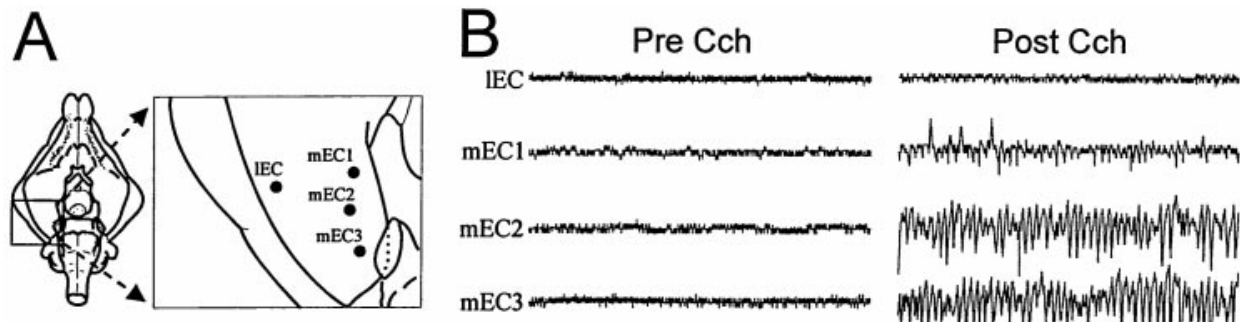

C

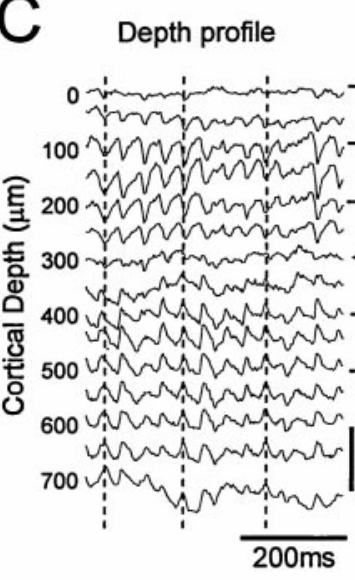

Power

Phase

Coherence

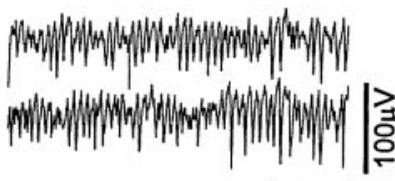

$\overline{500 \mathrm{~ms}}$

Figure 1. Carbachol-evoked $\gamma$ activity is generated in superficial layer II of mEC but not in IEC. A, Schematic diagram of a ventral view of the brain with an expansion of the entorhinal region showing the relative positions of recording electrodes. $B$, Extracellular field recordings from sites shown in $A$ before (left panel) and 30 min after arterial perfusion of $50 \mu \mathrm{M} \mathrm{CCh}$ (right panel). Continuous $\gamma$ activity was observed in the three mEC sites, but not in the IEC. $C$, Simultaneous recording of $\gamma$ activity at multiple depths in a different experiment using a 16 site linear silicon probe positioned in the $\mathrm{mEC}$ at a location between sites 2 and 3 in $A$. A reversal of the polarity of the oscillation was observed at a cortical depth of $300 \mu \mathrm{m}$. In the middle portion of panel $C$ the power, phase, and coherence of $\gamma$ frequency (25 $\mathrm{Hz})$ as a function of cortical depth is shown for the data illustrated in the leftmost panel. These values were obtained by averaging values in a $2 \mathrm{~Hz}$ frequency bandwidth surrounding the peak frequency of $\gamma$. The amplitude of the $\gamma$ signal was largest in superficial layers, with a minimum value at $300 \mu \mathrm{m}$, which corresponded to the location of a $180^{\circ}$ phase shift of the oscillation. Note that the coherence of $\gamma$ remained constant and elevated $(\sim 1.0)$ across all depths except at the site of reversal. The probe tract for this experiment is shown in the right-most panel in $C$. Note that the reversal point of the $\gamma$ oscillation (arrowhead) appears at the level of layer II.

careful removal, brains were submerged in a recording chamber filled with the same solution maintained at a temperature of $16^{\circ} \mathrm{C}$. Cerebral perfusion was achieved by cannulating the vertebral artery and perfusing through the existing brain vasculature at a rate of $5.5 \mathrm{ml} / \mathrm{min}$. Leaky vessels were ligated, and the brain was gradually $\left(0.2^{\circ} \mathrm{C} / \mathrm{min}\right)$ warmed to a final temperature of $32^{\circ} \mathrm{C}$ (unless otherwise specified). This protocol was reviewed and approved by the Committee on Animal Care and Use and by the Ethics Committee of the Istituto Nazionale Neurologico.

Extracellular field recordings were made with (1) glass micropipettes with a $10-\mu$ m-diameter tip and filled with a $\mathrm{NaCl}$ solution $(0.9 \%)$ in addition to any pharmacological agent (see below), (2) 16-site linear silicon probes $(50-100 \mu \mathrm{m}$ contact separation; kindly provided by Jamille Hetke of the Center for Neural Communication Technology, University of Michigan, Ann Arbor, MI), or (3) tungsten $4 \times 1$ matrix microelectrode arrays (410 $\mu \mathrm{m}$ tip separation; Frederick Haer Corporation, Bowdoinham, ME). All recordings, unless otherwise noted, were made at a depth of $500 \mu \mathrm{m}$ from the pial surface. Recordings at multiple sites were performed simultaneously. Signals amplified at a gain of 1000 using an AC amplifier (Biomedical Engineering, Thornwood, NY) were high-pass filtered at 0.2 $\mathrm{Hz}$ and low-pass filtered at $1000 \mathrm{~Hz}$. They were digitized on-line using customized software (Clampview) developed by G. Biella in collaboration with SIDeA (alliance member of National Instruments, Milan, Italy) and were stored on digital tape (DTR 2602 Biological, Claix, France) for off-line analysis. Single and dual channel spectral analysis was conducted off-line using Matlab (Mathworks, Natick, MA). After digitally filtering with a fourth order Butterworth function in a $2.5 \mathrm{~Hz}$ bandwidth around line frequency $(50 \mathrm{~Hz})$ to minimize coherence artifacts, the auto power, cross power, phase, and coherence spectra were computed for all signals and all signal pair combinations. We empirically determined by comparison of the same signals with and without filtering that this manipulation was adequate in eliminating line frequency artifacts without producing major alterations in the frequency band of interest which, in this study, was substantially lower than this $(25-30 \mathrm{~Hz})$. Stimulation of the lateral olfactory tract (LOT) was conducted with bipolar silver wires, insulated along their length except at the tips. Intracortical stimulation was conducted using thin insulated tungsten bipolar electrodes (Frederick Haer Corporation). In all preparations, before any experimental procedures, the response of the piriform or lateral entorhinal cortex to electrical stimulation of the LOT was assessed to verify the viability of the preparation. When metal electrodes were used for intracortical recording and stimulation, electrolytic lesions induced by $10 \mathrm{~mA}$ current application for 5-10 sec were made to mark the location of the electrode sites. Subsequently, the brains were fixed overnight with a $4 \%$ paraformaldehyde solution in a $0.1 \mathrm{M}$ sodium phosphate buffer and then sectioned by vibratome at a thickness of $100 \mu \mathrm{m}$. Sections were mounted, stained with thionin, and inspected for the location of electrode sites.

$\gamma$ activity was elicited in the $\mathrm{mEC}$ by either local pressure injection $(5-50$ $\mathrm{mM}$ in $0.9 \% \mathrm{NaCl}$ for $1-5 \mathrm{sec}$ ) or arterial perfusion $(25-100 \mu \mathrm{M}$ in perfusion solution) of CCh. As with microinjections of $\mathrm{CCh}$, injections of all other substances was conducted at a depth of $500 \mu \mathrm{m}$ using a modified recording pipette that allowed for simultaneous recording and drug injection. Other pharmacological agents used included the muscarinic antagonist atropine sulfate, the GABA receptor antagonist bicuculline methiodide (BMI), and the AMPA receptor antagonist 6-cyano-7-nitroquinoxaline-2,3-dione (CNQX) in concentrations ranging from 5-10 $\mu \mathrm{M}$ (arterial perfusion) and $0.1-5 \mathrm{~mm}$ (local injection). CNQX was predissolved in dimethylsulfoxide (DMSO) at a concentration of 5-25 mM before being added to the saline solution. Specifics of the diverse experimental procedures for intraparenchymal infusions can be found in Results.

All salts were obtained from BDH (Poole, UK) and all drugs from Sigma (St. Louis, MO), except CNQX, which was obtained from Tocris Cookson (Bristol, UK). Dextran was obtained from SIFR A (Isola della Scala, Italy).

\section{RESULTS}

As previously shown (van der Linden et al., 1999), either arterial perfusion $(25-100 \mu \mathrm{M})$ or local injections of CCh $(5-50 \mathrm{~mm})$ elicited fast oscillatory activity $(25 \pm 4 \mathrm{~Hz} ; n=18$; mean $\pm \mathrm{SD})$ that was localized to the mEC (Fig. $1 A, B$ ). In a subset of experiments $(n=5)$, the frequency of this activity increased from $29 \pm$ 3 to $45 \pm 2 \mathrm{~Hz}$ as the temperature of the preparation was raised to more physiological levels (from 30 to $36^{\circ} \mathrm{C}$; data not shown), suggesting that it most likely represents physiological $\gamma$ activity.

To characterize the specific layers responsible for the generation of this activity, a laminar profile analysis was undertaken. Recordings were made in the mEC using a 16-contact linear probe oriented orthogonally to the pial surface and therefore, to the laminar cortical arrangement of the mEC. The contacts were spaced evenly at an interlead distance of $50 \mu \mathrm{m}$ and spanned a total distance of $750 \mu \mathrm{m}$. Unlike laminar profiles performed by advancing a single electrode (van der Linden et al., 1999), recordings with multisite silicon probes enabled $\gamma$ activity to be recorded simultaneously at multiple depths in the same "column" of entorhinal tissue. The depth of the probe was regulated to leave one or two of the proximal channels outside the brain. In this way, the first proximal contact inside the brain was assumed to be at a depth of $50 \mu \mathrm{m}$. In all experiments $(n=5)$, a reversal of the raw $\gamma$ field potential rhythm appeared across the superficial layers of the mEC (Fig. $1 C$, arrow). These results were further analyzed by dual-channel spectral methods, using a comparison channel corresponding to the second-to-last contact on the probe itself as a reference for all other 


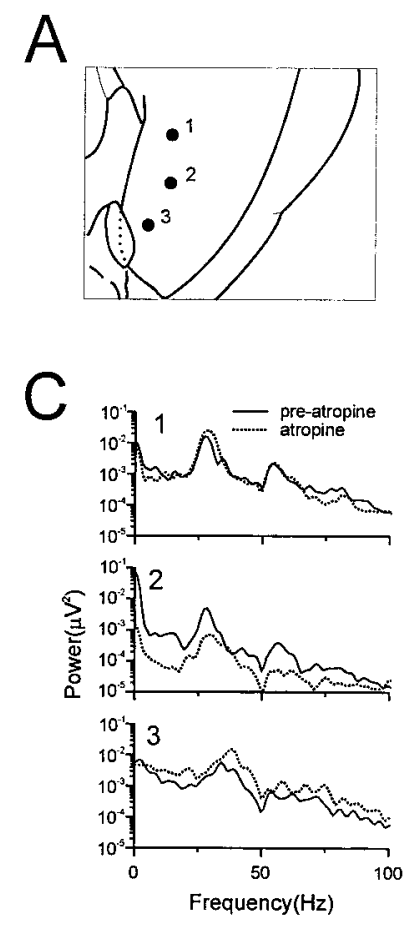

Figure 2. Local blockade of muscarinic transmission produces a localized inhibition of $\gamma$ activity. $A$, Expanded schematic diagram of the entorhinal region with recording electrode positions. $B$, Field recordings at positions noted in $A$ during $\gamma$ evoked by arterial perfusion with $100 \mu \mathrm{M}$ $\mathrm{CCh}$. The left panel shows traces directly before, during, and after a pressure pulse application of atropine sulfate through the recording pipette at position 2 (marked by the bar); the right panel shows the same series of recordings 5 min after atropine application. This manipulation evoked a robust inhibition of $\gamma$ only at the site in which the injection was made (expansion of the traces shown below). $C$, Power spectra of the signal before and 5 min after the atropine injection demonstrate a selective depression of the amplitude of the $\gamma$ signal in the recordings at position 2. Note different $\gamma$ frequency peaks at different sites. The "notch" appearing at $50 \mathrm{~Hz}$ in this and all other figures showing power spectra corresponds to the effects of digital filtering of the signals to eliminate line frequency noise (see Materials and Methods).

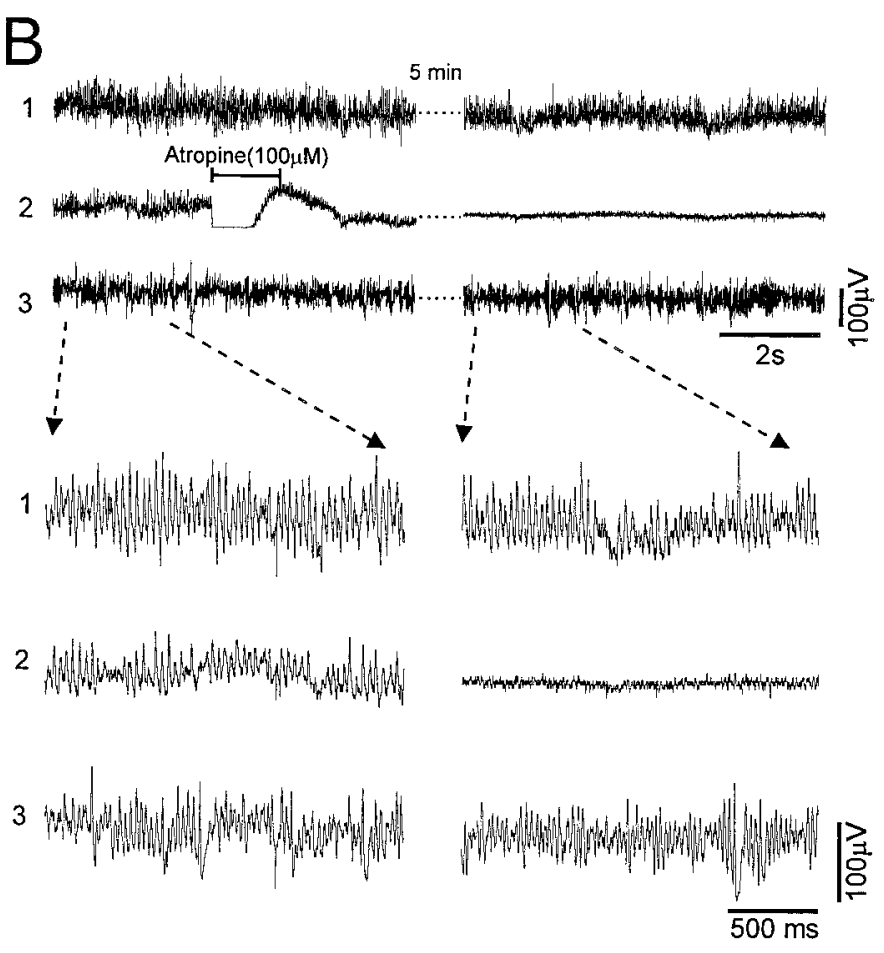

perfusion of atropine $(5 \mu \mathrm{m} ; n=4)$. Given the ability of local CCh application to evoke $\gamma$ activity (present results and van der Linden et al., 1999), muscarinic receptor excitation presumably takes place at the level of the mEC itself. This hypothesis was tested using pressure microinjections of atropine (100-500 $\mu \mathrm{M})$ in the mEC during $\gamma$ activity evoked by arterial perfusion of $\mathrm{CCh}$. As shown in Figure 2 and in all other experiments $(n=4)$, this manipulation inhibited $\gamma$ activity in the area directly surrounding the injection site as observed in both the recording traces (Fig. $2 B$ ) and power spectra (Fig. $2 C$ ) for site 2 . Such an inhibition was also observed without pressure ejection, when higher concentrations of atropine $(5 \mathrm{~mm})$ were allowed to freely diff use from recording pipettes $(n=$ 2). Therefore, local muscarinic excitation appears to provide the driving force for the elicitation of $\gamma$ activity by CCh in the mEC. In contrast to arterial perfusions of atropine $(5 \mu \mathrm{M})$ (van der Linden et al., 1999), the effect of intraparenchymal application of higher concentrations of atropine did not reverse, even after wash times as long as $2 \mathrm{hr}$.

Previous studies have proposed that $\gamma$ activity depends on the rhythmic synchronization of IPSPs on principal neurons (Whittington et al., 1995; Buhl et al., 1998; Fisahn et al., 1998; Penttonen et al., 1998). Blocking fast GABAergic neurotransmission with the receptor antagonist BMI tested this hypothesis. After the elicitation of $\gamma$ by arterial perfusion of 25-50 $\mu \mathrm{M} \mathrm{CCh}$, coperfusion of BMI $(10 \mu \mathrm{M})$ resulted in the abolition of the fast rhythm evoked by CCh $(n=2)$. However, systemic blockade of GABAergic neurotransmission also evoked large-amplitude epileptiform activity (cf. Librizzi and de Curtis, 1999), which could have masked $\gamma$ activity through other, nonspecific mechanisms such as postictal or spreading depression. Therefore, brief microinjections of BMI were made directly within the mEC during $\gamma$ produced by arterial perfusion of CCh (Fig. 3B, left traces; $n=6$ ). This manipulation completely abolished $\gamma$ activity as observed both in the recording traces (Fig. $3 B$ ) and power spectra (Fig. $3 C$ ). Similarly to the effects of locally applied atropine, this blockade occurred in a spatially localized region directly surrounding the injection site. To test whether the microinjection procedure per se interfered with the production of $\gamma$, similar pressure microinjections of saline solution were made $(n=3)$. As illustrated in Figure $3 B$ (right panel), after the washout

of BMI effect, a pressure injection of saline from electrode 2 had no evoked $\gamma$ activity was found to be dependent on the activation of muscarinic receptors because it was completely blocked by arterial 


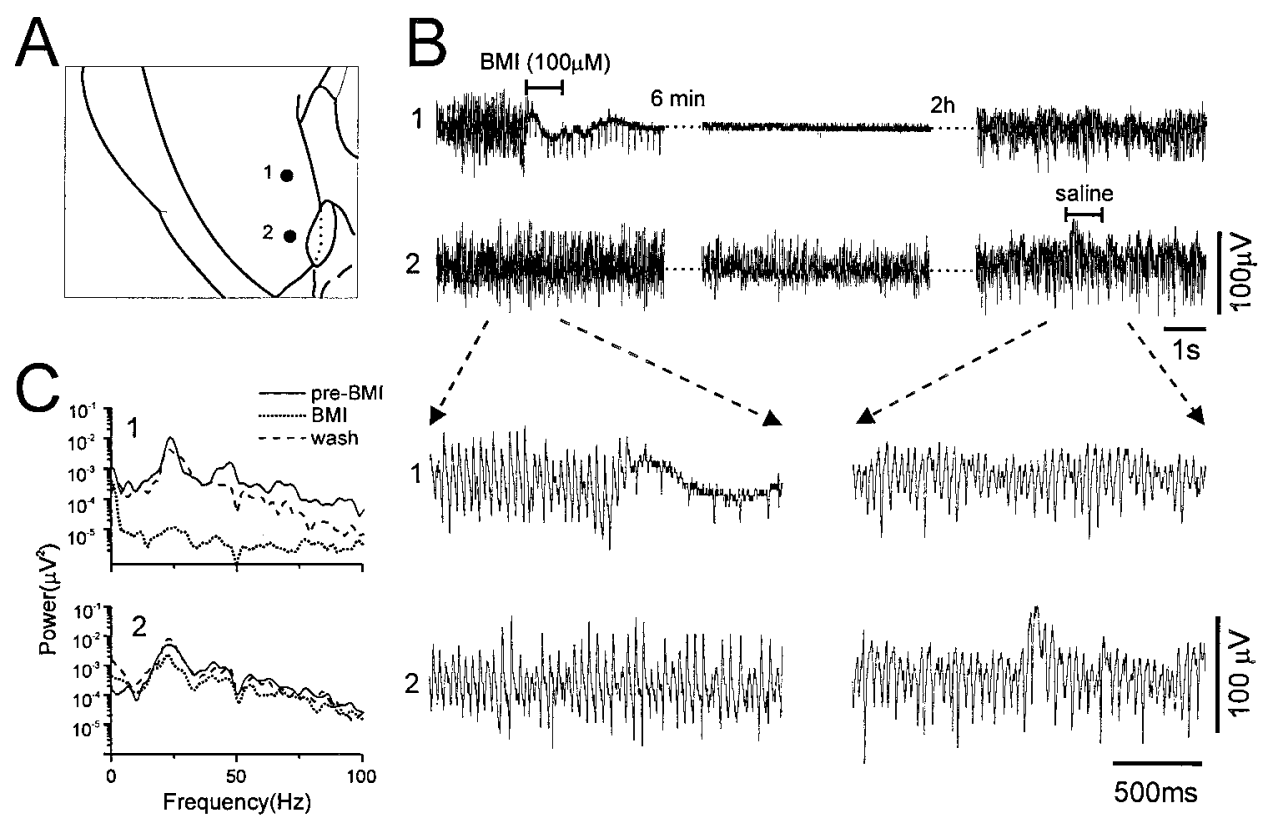

Figure 3. Local blockade of fast GABAergic transmission produces a localized abolition of $\gamma$ activity. $A$, Schematic diagram of the recording electrode sites. $B$, Field recordings at positions noted in $A$ during $\gamma$ activity evoked by arterial perfusion with $50 \mu \mathrm{M}$ CCh with expansions of selected portions shown below. The left panel shows the recordings before, during, and directly after a pressure pulse application of BMI through the recording pipette at position 1 (see bar). A selective abolition of $\gamma$ activity is observed at the same site at which the injection was made (middle panel). This effect was reversible after a $2 \mathrm{hr}$ wash period (right panel). After the washout of bicuculline, a similar pressure pulse injection of saline through the recording pipette at site 2 (see bar, right panel) had no influence on $\gamma$ activity at any site. $C$, Power spectra of the signal before, $6 \mathrm{~min}$, and $2 \mathrm{hr}$ after BMI application demonstrate a reversible abolition of the amplitude of the $\gamma$ signal in the recordings at position 1 . effect on $\gamma$ activity. Thus, the suppressive effect seen with drug application was specific to its pharmacological action.

Because fast glutamatergic transmission has been shown to be important in the generation of CCh-induced $\gamma$ activity in hippocampal and neocortical slices (Buhl et al., 1998; Fisahn et al., 1998) we also performed brief local pressure applications of CNQX (5 mM) during $\gamma$ activity evoked by arterial perfusions of CCh. In all experiments performed $(n=6)$, and as illustrated in Figure 4, a marked depression of the amplitude of $\gamma$ was observed, again in a spatially localized region surrounding the injection pipette. Likewise, a depression of the potential evoked by electrical stimulation of associative fibers was seen at the same, but not at distant sites in the mEC (Fig. 4C, right panel). The depression of both $\gamma$ and the evoked potential was partially recovered in three cases over a washout period of 30-90 min. In contrast, infusions of saline and vehicle (DMSO; $n=3$ ), as well as lower concentrations of CNQX $(500 \mu \mathrm{M} ; n=2)$, had no effect upon $\gamma$ activity (data not shown). Therefore, it would appear that as for $\gamma$ activity in brain slices, the excitatory network contributes to the generation of carbacholgenerated $\gamma$ activity in the mEC.

The spatial restriction of the pharmacological effects described above suggests the existence of multiple independent generators of $\gamma$ activity across the planar horizontal extent of mEC superficial layers. Further support for this idea was garnered when recordings were made using electrodes with a high $(100 \mathrm{~mm})$ concentration of CCh. Soon (1-5 min) after pipette placement at a depth of $500 \mu \mathrm{m}$ in the mEC (but not the lEC; $n=2$ ), $\gamma$ developed in a highly circumscribed region of $\sim 500 \mu \mathrm{m}$ surrounding the electrode site (Fig. $5 B ; n=10$ ). This was confirmed by changing the position of another recording electrode (without $\mathrm{CCh}$ ) from a distant site to a closer site from position 1 a to 1 b (Fig. 5B). This effect was most likely mediated through passive diffusion of $\mathrm{CCh}$ into the $\mathrm{mEC}$ because $\gamma$ activity disappeared shortly after $(5-10 \mathrm{~min})$ removal of the CCh-filled electrode (Fig. 5B, right panel; $n=3$ ). $\gamma$ evoked in this manner was abolished by arterial perfusion of atropine $(5 \mu \mathrm{M}$, $n=2$; data not shown). Sequential recordings taken at multiple depths at a site proximal to the CCh-filled electrode demonstrated a phase reversal at $\sim 300 \mu \mathrm{m}$, confirming its similarity to $\gamma$ activity evoked by arterial perfusion ( $n=2$; data not shown). When two pipettes containing $100 \mathrm{~mm} \mathrm{CCh}$ were placed in the $\mathrm{mEC}$ (Fig. 5C), $\gamma$ was generated at both sites (Fig. $5 D$ ). When a large distance separated these electrodes $(>1 \mathrm{~mm})$ the coherence of the $\gamma$ signals across both was extremely low (near random levels; Fig. $5 E$, dotted line). In fact, the peak frequency of $\gamma$ was often different between the two sites. When the separation between distally spaced elec- trodes was reduced by moving one electrode closer to the other, the coherence of $\gamma$ increased dramatically (Fig. $5 D, E$; continuous line, $n=5)$.

In light of the spatial localization observed with focal pharmacological applications, we elected to study the horizontal spatial distribution and synchronization of $\gamma$ activity evoked by arterial perfusion of $\mathrm{CCh}$ by performing multisite recordings across the surface of the mEC. This was conducted with two electrode arrays, each having four contacts with interelectrode distances of $410 \mu \mathrm{m}$ between adjacent pairs within an array (Fig. 6A). After the induction of $\gamma$ (Fig. $6 B)$, dual-channel spectral analysis was conducted for every pair of electrodes both within and between arrays. These data are illustrated in Figure $6 C$. Linear coherence was calculated at the peak frequency corresponding to the $\gamma$ peak in the cross spectra power peak. As shown in Figure $6 D$ and in all other experiments, there was a consistent and significant $(p<0.01)$ negative linear relationship between coherence and the interrecording site distances (Fig. 6D). Across all experiments, this relationship was characterized by the following equation:

coherence $=1.0-0.44$ (intersite distance in millimeters)

$$
(r=-0.995 ; p<0.01 ; n=5) .
$$

However, given the variation in signal amplitude across electrode locations, a possible confounding element to the coherence measurements could have been caused by the differences in relative amplitudes of the signals compared. Therefore, a regression analysis was conducted between the $\gamma$ cross-spectral amplitude (an indication of the relative amplitude of the $\gamma$ signal between two sites) and $\gamma$ coherence for each pair of signals. No significant linear relationship between these two parameters was observed (Fig. $6 E$; $p>0.01 ; n=5)$. Thus, $\gamma$ coherence appeared to be independent of the signal amplitudes measured in this study.

To characterize the time course of synchronization between different sites across time as $\gamma$ activity developed during arterial perfusion of $\mathrm{CCh}$, coherence analysis was performed at multiple time points, before, during and after the appearance of $\gamma$ activity in different experiments $(n=5)$. As illustrated in Figure $7 B$, the amplitude of $\gamma$ activity was typically low at onset and gradually increased with time. Figure $7 C$ demonstrates that, despite variation in cross-spectral power amplitude for $\gamma$ activity with time, $\gamma$ coherence remained stable for given electrode pairs (far and near pairs illustrated in the left and right panels, respectively). The coherence measurements obtained in five experiments between near and far recording sites showed no significant changes across time (see 
Figure 4. Local blockade of fast glutamatergic transmission produces a localized inhibition of $\gamma$ activity. $A$, Schematic diagram of the recording and stimulation electrode sites. $B$, Field recordings at positions noted in $A$ during $\gamma$ evoked by arterial perfusion with $50 \mu \mathrm{M}$ CCh. The left panel shows the recordings directly before, during, and after a pressure pulse application (see bar) of CNQX through the recording pipette at position 1 , and the middle panel shows the same series of recordings $5 \mathrm{~min}$ after the application. This manipulation evoked a robust inhibition of $\gamma$ only at the site in which the injection was made (expansion of the traces shown below). Correspondingly, in the right panel, the potentials evoked by stimulation of superficial associational fibers before (continuous lines) and $7 \mathrm{~min}$ after the local application of CNQX (dotted lines) show a selective depression only at site 1 . $C$, Power spectra of the field signals before and $5 \mathrm{~min}$ after the injection demonstrate a selective depression of the amplitude of the $\gamma$ signal in the recordings at position 1 .

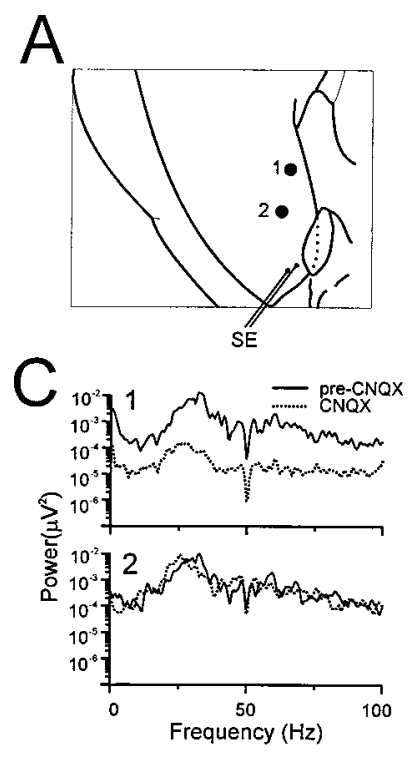

B
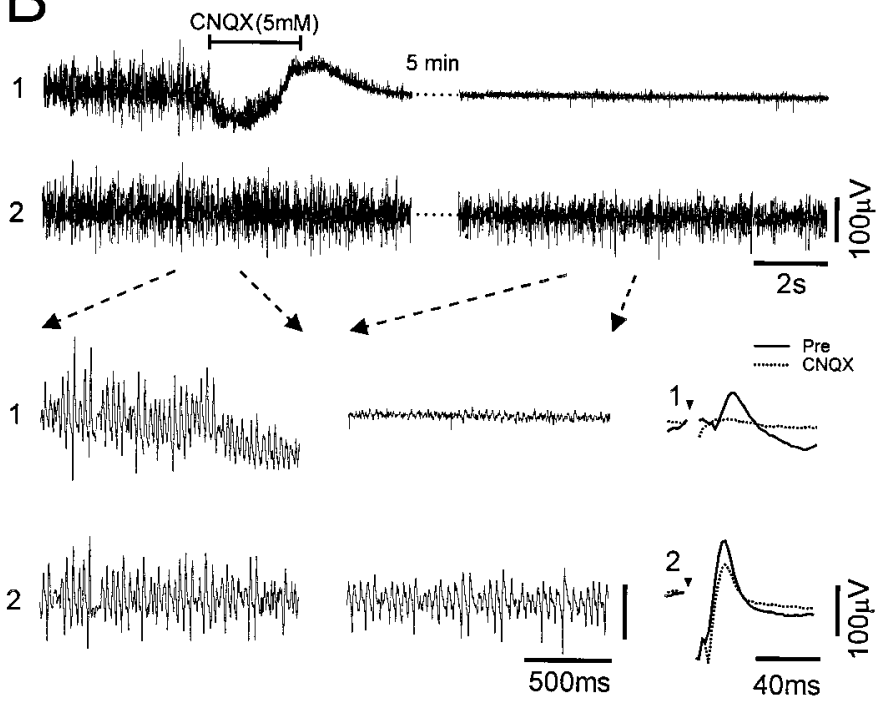

regression fittings in Fig. $7 D ; p>0.01 ; n=5)$. This provides further evidence that $\gamma$ synchronization depends on the spatial relationship between the sites, rather than on the amplitude of $\gamma$ activity.

As previously reported (van der Linden et al., 1999), both perfusion and local injection of CCh occasionally induced transient trains of theta-like oscillations in the $\mathrm{mEC}$, similar in form to activity induced by $\mathrm{CCh}$ in the $\mathrm{mEC}$ slice preparation (Dickson and Alonso, 1997). Isolated theta sequences were composed of rhythmic waves between 3 and $8 \mathrm{~Hz}$ that appeared simultaneously across the surface of the mEC (Fig. 8B, left traces) and often preceded the onset of a stable $\gamma$ oscillatory activity (Fig. $8 B$, right traces). In contrast to $\gamma$ coherence measured in the same experiment, theta coherence showed little variation with intersite distance (Fig. 8C, compare left and right panels for theta and $\gamma$, respectively). In fact, as illustrated in Figure $8 D$, theta-like oscillations in different experiments were almost perfectly coherent (average value of $0.93 \pm 0.04, n=5$ ) across the covered extent of the mEC (open squares), whereas coherence values of $\gamma$ decreased with distance between recording sites (filled squares). There was a significant negative linear relationship between coherence and distance for $\gamma(r=-0.995 ; p<0.01 ; n=5)$ but not for theta $(r=$ $-0.408 ; p>0.01 ; n=5)$. Thus, the spatial limitation of $\gamma$ coherence would appear to reflect an unique property of $\gamma$ activity itself.

\section{DISCUSSION}

Based on our pharmacological experiments, we hypothesize that CCh-evoked $\gamma$ activity in the mEC involves an interaction between reciprocally connected inhibitory interneurons and principal cells in superficial layers. Previous studies on the mechanisms of $\gamma$ activity generation have proposed that the extracellular field oscillation reflects the bombardment of rhythmic and synchronized IPSPs on principal neurons (Whittington et al., 1995; Buhl et al., 1998; Fisahn et al., 1998; Penttonen et al., 1998) (for review, see Buzsáki and Chrobak, 1995; Jefferys et al., 1996; Connors and Amitai, 1997; Ritz and Sejnowski, 1997). The demonstration that $\gamma$ activity in our preparation was crucially dependent on the functional preservation of fast GABAergic neurotransmission is consistent with this idea. Synchronization within an inhibitory interneuronal network has been shown to be mediated either through synaptic (Whittington et al., 1995; Traub et al., 1996; Wang and Buzsaki, 1996), or gap junction connections between interneurons (Galarreta and Hestrin, 1999; Gibson et al., 1999; Mann-Metzer and Yarom, 1999; Fukuda, 2000) or both (Tamás et al., 2000). Although independent synchronization of an inhibitory interneuronal network has been suggested to be sufficient in sustaining $\gamma$ oscillations (Whittington et al., 1995; Traub et al., 1996; Wang and Buzsaki, 1996), our present findings concerning the dependence of
Figure 5. Diffusion of carbachol from recording pipettes evokes spatially localized $\gamma$ activity. $A$, Schematic diagram of the recording electrode positions. As noted, the pipette used for recording at site 2 contained $100 \mathrm{~mm}$ CCh and electrode 1 was moved from position 1a to position 1b. $B$, Field activity recorded at distant (position 1a, left panel) and adjacent (position $1 b$, middle panel ) separations of the recording electrodes and $10 \mathrm{~min}$ after the removal of the CCh electrode (right pan$e l) . \gamma$ activity was observed with the saline pipette only when it was positioned in close proximity to the CCh pipette (left and middle panels, respectively) and dissipated shortly after removal of the $\mathrm{CCh}$ pipette (right panel). C, Diagrammatic representation of an experiment using two CCh-filled electrodes. As noted, electrode 1 was moved from position 1a to position 1 b. $D$, Field recordings at distant (left panel) and near (right panel) sites. $\gamma$
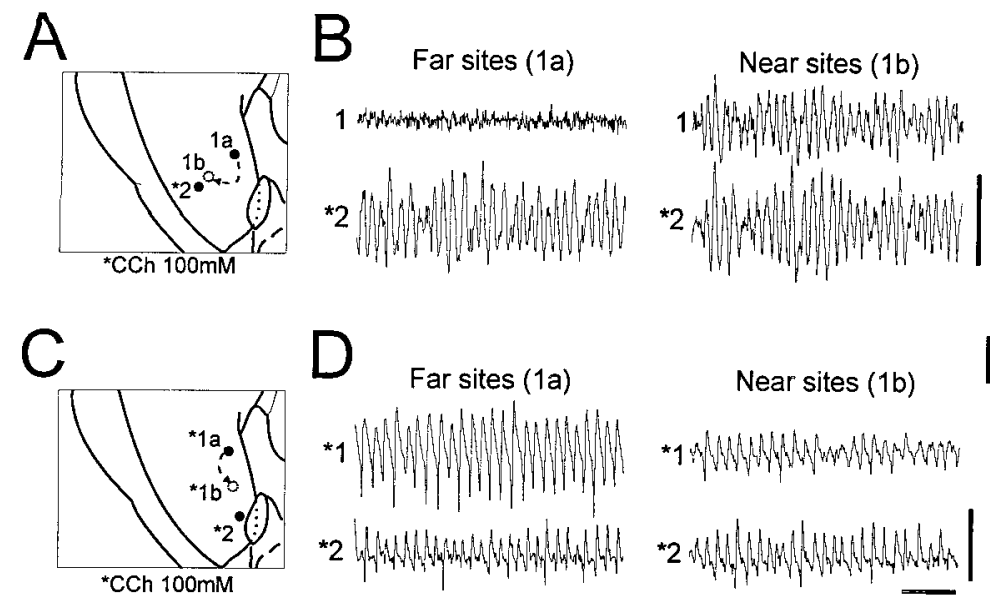

Electrode 2 removed

was recorded from both electrodes at both positions. $E$, Coherence spectrum of the signals shown in $D$. Although $\gamma$ amplitude was high for both placements, the coherence at $\gamma$ frequencies (arrow) was negligible for far separations (continuous line) as compared to near separations (dotted line). 

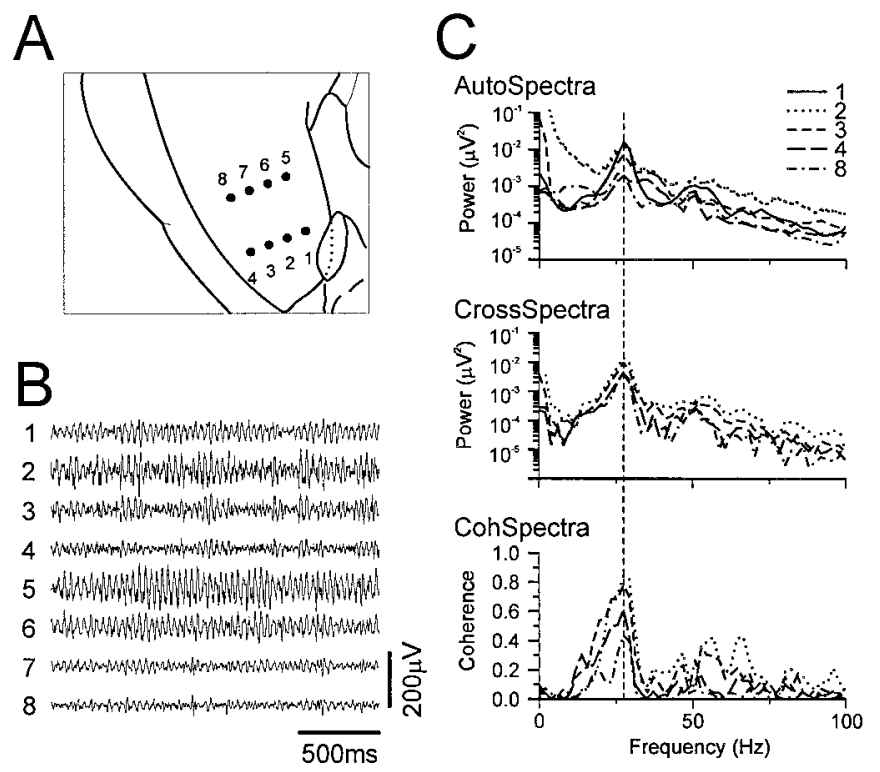

D

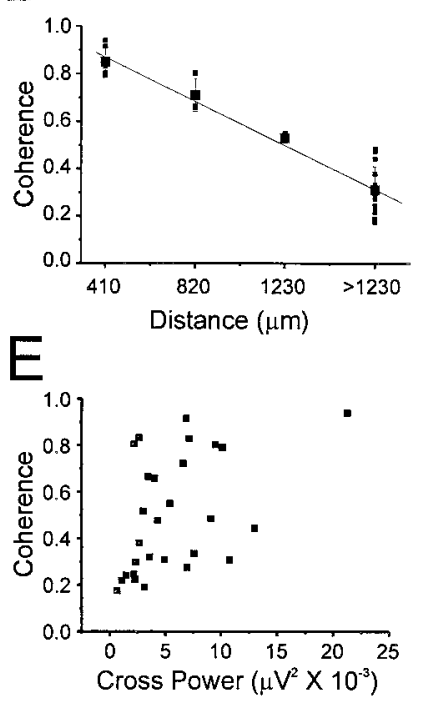

Figure 6. $\quad \gamma$ activity evoked by arterial perfusion of CCh shows spatially limited synchronization across the surface of the EC. $A$, Schematic diagram of the two recording array positions. $B, \gamma$ field recordings from all eight sites after arterial perfusion with $50 \mu \mathrm{m}$ CCh. $C$, Auto, cross power, and coherence spectrum for signals at sites $2,3,4$, and 8 as compared to site 1 . The coherence values at the $\gamma$ frequency peak (vertical dotted line) decreased as the distance between electrode sites increased. $D$, Plot of $\gamma$ coherence values as a function of interelectrode distance for all possible pairwise comparisons in the experiment illustrated in $B$. The regression of the average coherence (large squares) on distance demonstrated a significant negative linear relationship of the equation: coherence $=1.0-0.45$ (interelectrode distance in millimeters) $(r=$ $-0.995 ; p<0.01)$. E. Plot of $\gamma$ coherence as a function of relative $\gamma$ amplitude (cross power at $\gamma$ frequency) for all possible pairwise comparisons for the same data as in $B$. The regression of coherence on cross power revealed no significant linear relationship $(p>0.01)$. $\gamma$ activity on fast glutamatergic transmission support the recently reported conclusion that recurrent glutamatergic excitation is necessary for $\gamma$ generation (Buhl et al., 1998; Fisahn et al., 1998; Leung, 1998). Anatomical studies have shown that layer II principal neurons form autoassociative recurrent contacts within layer II (Kohler, 1986; Klink and Alonso, 1997a; Dolorfo and Amaral, 1998), and physiological evidence has demonstrated robust feedforward and recurrent inhibitory mechanisms in principal neurons within this layer (Jones and Buhl, 1993; Jones, 1994). Moreover, inhibitory inputs have been shown to reset discharge in principal neurons of the mEC layer II (Dickson et al., 2000) and hippocampus (Cobb et al., 1995; Chapman and Lacaille, 1999). Therefore, it is likely that the recurrent excitatory population discharge of mEC layer II principal cells is entrained at $\gamma$ frequencies by rhythmic IPSPs. In support of this, we have occasionally observed single-unit activity in superficial layers showing a phase relation to the extracellular $\gamma$ rhythm (cf. Chrobak and Buzsaki, 1998a).

A novel finding of the present study is that the basic mEC circuit responsible for muscarinic-dependent $\gamma$ activity is highly localized. Whereas this may not seem surprising based on the finding that $\gamma$ activity can be observed in slice preparations in which horizontal connectivity is highly limited (to often $<400 \mu \mathrm{m}$ ) (cf. Whittington et al., 1995; Buhl et al., 1998; Fisahn et al., 1998), what is surprising is that despite the fact that horizontal connectivity in the whole brain preparation is intact, this spatial specificity of $\gamma$ generation appears to be preserved. The rhythmic synchronization of neuronal activity at $\gamma$ frequencies in spatially localized regions over the surface of the mEC may confer a functional modular organization to this structure, in the absence of a typical cortical columnar arrangement. This conclusion is supported by the following experimental evidence: (1) local application of pharmacological agents impaired the generation of $\gamma$ oscillations in restricted portions of the mEC, (2) $\gamma$ activity could be induced in highly localized patches of cortex restricted to the immediate zone surrounding a CChcontaining electrode, (3) $\gamma$ oscillations induced by two CChcontaining electrodes at remote positions showed little coherence
A

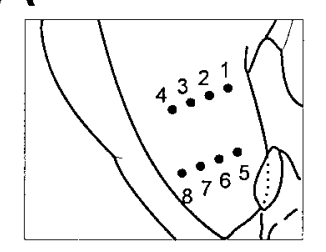

B
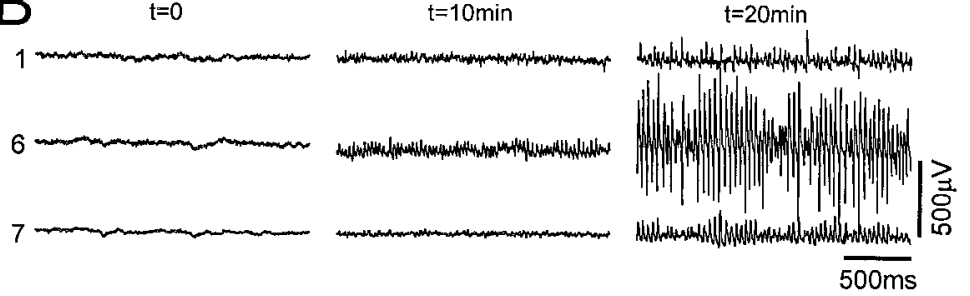

C

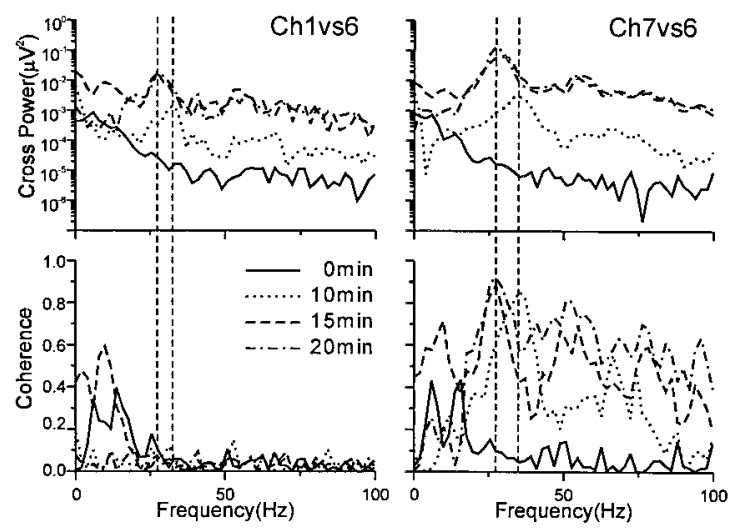

Figure 7. $\gamma$ synchronization between given sites remains consistent across time, independently of signal amplitude. $A$, Schematic diagram of the recording array positions. $B$, Field recordings from sites 1,6 , and 7, demonstrating development of $\gamma$ at different times during perfusion of $50 \mu \mathrm{M}$ CCh. $\gamma$ was initially observed $10 \mathrm{~min}$ after the start of $\mathrm{CCh}$ perf usion. $C$, Cross and coherence spectra for channels 1 and 6 (far separation) and channels 6 and 7 (near separation) during different times after the start of $\mathrm{CCh}$ perfusion $(t=0$, continuous line; $t=10 \mathrm{~min}$, dotted line; $t=15 \mathrm{~min}$, dashed line; $t=20$, dash-dot line). Despite differences in amplitude of the $\gamma$ signal across time, the coherence of $\gamma$ remained constant within the near and far site comparisons. $D$, Scatter plot and regression fittings of $\gamma$ coherence for near $(410 \mu \mathrm{m}$; filled shapes-continuous lines) and far (>1230 $\mu \mathrm{m}$; open shapesdotted lines) electrode separations as a function of time. Different experiments are represented by different geometrical shapes. Coherence values were consistently high for near electrodes and consistently low for far electrodes from the onset of $\gamma$. No regression fitting for any experiment showed a significant linear trend $(p>0.01)$. 
A

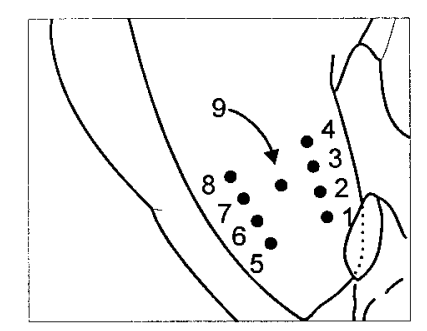

Figure 8. Spatial synchronization is specific for $\gamma$, but not theta-like activity elicited by CCh. $A$, Schematic diagram of recording array positions. $B$, Field recordings from all electrode sites demonstrating theta-like (left) and $\gamma$ (right) oscillatory activity that developed after an injection of $5 \mathrm{~mm} \mathrm{CCh}$ at location 9. $C$, Cross and coherence spectra for a series of electrode comparisons to channel 4 during both theta (left) and $\gamma$ (right). Note that the coherence values at theta frequencies remained high regardless of interelectrode distance, whereas $\gamma$ coherence decreased with increasing interelectrode distances. $D$, Scatter plot of theta (empty squares) and $\gamma$ (filled squares) coherence as a function of interelectrode distance. $\gamma$ coherence showed a significant negative linear relationship with distance $(r=-0.995$; $p<0.01)$. Theta coherence, however, was uniformly high, and independent of distance and showed no significant linear trend $(r=-0.408 ; p>0.01)$.
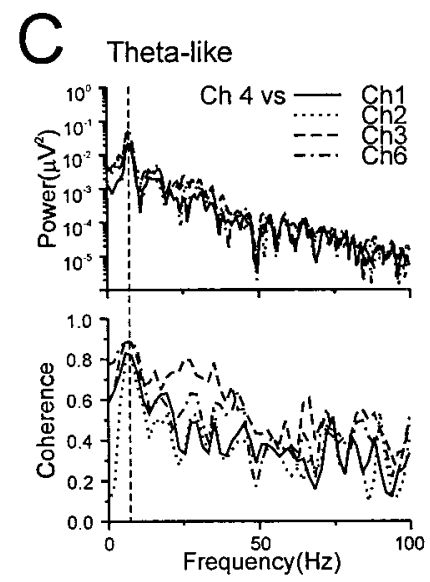
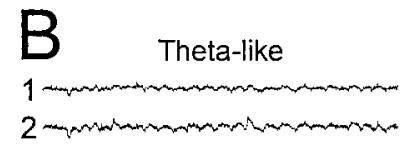

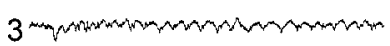

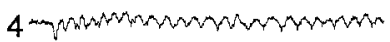

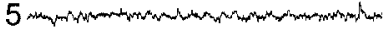

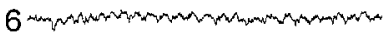

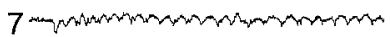

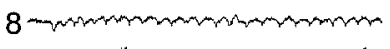
9 mand $2 s$

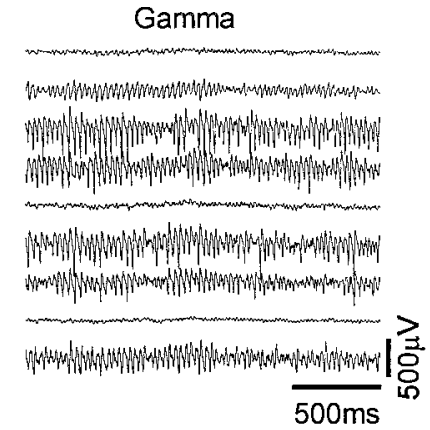

Gamma

D

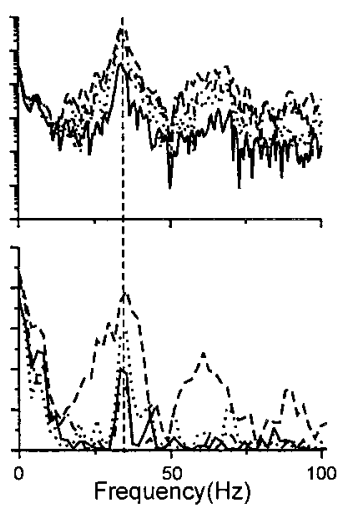

(synchronization), and (4) the synchronization of $\gamma$ activity at different mEC locations showed a strict linear decrease with distance, whereas $\gamma$ activity in a "column" of mEC tissue as recorded using a multidepth probe was consistently and highly coherent. This relationship was a unique property of $\gamma$ activity because, in the same experiments, theta-like activity showed high synchronization across the mEC surface, regardless of the interelectrode distance.

Estimations of the minimal size of cortical tissue necessary for the generation of $\gamma$ activity (corresponding to a "functional module") can be garnered from the evaluation of the linear relationship between $\gamma$ coherence and distance. Because coherence values are mathematically analogous to the correlation coefficient, the squared value of coherence describes the proportion of variance predicted in one signal by the other, i.e., for perfect correspondence coherence ${ }^{2}=1$ (Bendat and Piersol, 1986; Armitage and Berry, 1994). Therefore, if we assume that high synchronization occurs with coherence values of at least 0.75 (corresponding to a minimum relationship between signals of $0.75^{2} * 100=56 \%$ ), it can be estimated that the upper limit for the radius of the basic cortical area that generates independently $\gamma$ oscillations is 500-600 $\mu \mathrm{m}$, according to the regression equation elaborated in Results. This value may be substantially smaller, given our findings with diffusion of $\mathrm{CCh}$ from pipette tips. Similarly, in the mEC slice preparation, it has been shown that $\mathrm{CCh}$ induces a nonglutamatergic-dependent synchronization of layer II interneurons in spatially localized "pools" having a radial distance of $\sim 250-300 \mu \mathrm{m}$ (Dickson and Alonso, 1997). Interestingly, our identification of a limited cortical element in which $\gamma$ coherence was found to be maximal (i.e., a functional mEC module) corresponds well with the observation of highly synchronous fast oscillations within orientation columns of the visual cortex (Eckhorn et al., 1988; Gray et al., 1989; Gray and Singer, 1989). It is therefore tempting to speculate that these functional modules corresponding to separate and independent generators of $\gamma$ activity represent a fundamental hardware processing unit in the mEC.

Interactions between mEC functional modules might be performed by tangential associational connections within layer II of the mEC (cf. Gray et al., 1989). As previously discussed, these connections may be mediated through either or both excitatory and inhibitory connections. Anatomical findings have shown a robust collateralization of axonal branches spreading in a tangential plane of both excitatory (Kohler, 1986; Klink and Alonso, 1997a; Dolorfo and Amaral, 1998) and inhibitory (GABAergic) (Jones and Buhl, 1993; Wouterlood et al., 1995) neurons within EC layer II. A prevalent contribution of inhibitory collaterals in connecting functional modules was indicated by the difference in the efficacy of locally applied BMI versus CNQX. Indeed, whereas $\gamma$ activity was completely abolished by GABAa receptor antagonism, AMPA receptor blockade, although completely eliminating the stimulusevoked field potential and substantially reducing the amplitude of the oscillation, did not eliminate the $\gamma$ spectral peak completely. A physiological mechanism by which coherence of $\gamma$ activity across extremely distant modules within the mEC may occur is via an extrinsic synchronizing input, such as from the medial septum, which has been shown to be important for the regulation of theta rhythmic activity in the mEC (Mitchell et al., 1982; Dickson et al., 1995; Jeffery et al., 1995) and both theta and $\gamma$ in the hippocampus (Bland, 1986; Ma and Leung, 1999). Indeed, there may exist the possibility that the slow (theta) rhythm itself exerts a synchronizing influence on the superimposed $\gamma$ oscillation (cf. Buzsáki and Chrobak, 1995).

Neocortical $\gamma$ oscillations, through the synchronization of neuronal activity on a fast time scale (tens of milliseconds) have been shown to be a mechanism for the temporal formation of ensembles that code for both simple and complex aspects of sensory stimuli (for review, see Gray, 1994; Singer and Gray, 1995; Laurent, 1996; Connors and Amitai, 1997; Basar et al., 1999; Tallon-Baudry and Bertrand, 1999). This synchronization is enhanced with manipulations that induce cortical or behavioral arousal, typically through activation of ascending cholinergic projections (Steriade et al., 1991; Metherate et al., 1992; Munk et al., 1996; Cape and Jones, 1997; Herculano-Houzel et al., 1999) that may correlate with attentional processes (Tiitinen et al., 1993; Maloney et al., 1996). Accordingly, $\gamma$ activity in the EC has been shown to be modulated in a similar way across behavioral and sleep-wake states (Chrobak and Buzsáki, 1998b). Because the EC is considered to represent a 
supramodal associational cortical region in which inputs from multiple sensory areas converge (Van Hoesen, 1982; Amaral and Witter, 1989; Witter et al., 1989), a state-dependent regulation of $\gamma$ synchronization within and between spatial modules could set a condition that facilitates or impedes binding between different sensory stimuli. $\gamma$ activity is generated by layer II, which receives associative neocortical inputs and is itself the source of the perforant path input to the hippocampus (Steward and Scoville, 1976). The existence of a regulatory function mediated by $\gamma$ synchronization in such a nodal element in the cortical-hippocampal system could be crucial for information processing, storage, and retrieval in limbic memory circuitry (cf. Buzsáki and Chrobak, 1995).

In summary, the present study demonstrates the uniqueness and advantages of the isolated whole brain preparation for recording and comparing activity within and across brain regions with a precision, stability, and spatial definition otherwise impossible to attain in either in vivo or in vitro conditions. Muscarinic activation, at the level of the $\mathrm{mEC}$ in this preparation, provides a good model for studying the generation of $\gamma$ activity because of its close correspondence to that described in vivo (Chrobak and Buzsáki, 1998b). Our findings suggest that $\gamma$ activity synchronizes activity in spatially localized cortical modules and is sustained by a circuit involving interactions between inhibitory and excitatory neurons within mEC layer II. We propose that such functional modules in the $\mathrm{mEC}$ represent a fundamental organizing device that constrains the processing and throughput of information in limbic circuitry.

\section{REFERENCES}

Ahissar E, Vaadia E, Ahissar M, Bergman H, Arieli A, Abeles M (1992) Dependence of cortical plasticity on correlated activity of single neurons and on behavioral context. Science 257:1412-1415.

Alonso A, García-Austt E (1987) Neuronal sources of theta rhythm in the entorhinal cortex of the rat. II. Phase relations between unit discharges and theta field potentials. Exp Brain Res 67:502-509.

Alonso A, Köhler C (1984) A study of the reciprocal connections between the septum and the entorhinal area using anterograde and retrograde axonal transport methods in the rat brain. J Comp Neurol 225:327-343.

Alonso A, Llinás RR (1989) Subthreshold $\mathrm{Na}^{+}$-dependent theta-like rhythmicity in stellate cells of entorhinal cortex layer II. Nature 342:175-177.

Amaral DG, Witter MP (1989) The three-dimensional organization of the hippocampal formation: a review of anatomical data. Neuroscience 31:571-591.

Armitage P, Berry G (1994) Statistical methods in medical research, Ed 3. Oxford: Blackwell Scientific.

Basar E, Basar-Eroglu C, Karakas S, Schurmann M (1999) Oscillatory brain theory: a new trend in neuroscience. IEEE Eng Med Biol Mag 18:56-66.

Bendat JS, Piersol AG (1986) Random data: analysis and measurement procedures, Ed 2. New York: Wiley.

Bland BH (1986) The physiology and pharmacology of hippocampal formation theta rhythms. Prog Neurobiol 26:1-54.

Bragin A, Jandó G, Nádasdy Z, Hetke J, Wise K, Buzsáki G (1995) Gamma $(40-100 \mathrm{~Hz})$ oscillation in the hippocampus of the behaving rat J Neurosci 15:47-60.

Buhl EH, Tamás G, Fisahn A (1998) Cholinergic activation and tonic excitation induce persistent gamma oscillations in mouse somatosensory cortex in vitro. J Physiol (Lond) 513(Pt 1):117-126.

Buzsáki G (1989) Two-stage model of memory trace formation: a role for "noisy" brain states. Neurosci 31:551-570.

Buzsáki G, Chrobak JJ (1995) Temporal structure in spatially organized neuronal ensembles: a role for interneuronal networks. Curr Opin Neurobiol 5:504-510

Cape E, Jones B (1997) Modulation of sleep-wake state and cortical activity following injections of agonists into the region of cholinergic basal forebrain neurons. J Neurosci 18:2653-2666.

Chapman CA, Lacaille J-C (1999) Cholinergic induction of thetafrequency oscillations in hippocampal inhibitory interneurons and pacing of pyramidal cell firing. J Neurosci 19:8637-8645.

Chrobak JJ, Buzsáki G (1998a) Gamma oscillations in the entorhinal cortex of the freely behaving rat. J Neurosci 18:388-398.

Chrobak JJ, Buzsáki G (1998b) Operational dynamics in the hippocampal entorhinal axis. Neurosci Biobehav Rev 22:303-310.

Cobb SR, Buhl EH, Halasy K, Paulsen O, Somogyi P (1995) Synchronization of neuronal activity in hippocampus by individual GABAergic interneurons. Nature 378:75-78.

Connors BW, Amitai Y (1997) Making waves in the neocortex. Neuron 18:347-349.

de Curtis M, Pare D, Llinás RR (1991) The electrophysiology of the olfactory-hippocampal circuit in the isolated and perfused adult mammalian brain in vitro. Hippocampus 1:341-354. de Curtis M, Biella G, Buccellati C, Folco G (1998) Simultaneous investigation of the neuronal and vascular compartments in the guinea pig brain isolated in vitro. Brain Res Brain Res Protoc 3:221-228.

de Curtis M, Dickson C, Panzica F, Pizzi R (1999) Pharmacology and organization of carbachol-induced gamma activity in the medial entorhinal cortex. Soc Neurosci Abstr 29:903.

Dickson CT, Alonso A (1997) Muscarinic induction of synchronous population activity in the entorhinal cortex. J Neurosci 17:6729-6744.

Dickson CT, Kirk IJ, Oddie SD, Bland BH (1995) Classification of thetarelated cells in the entorhinal cortex: cell discharges are controlled by the ascending brainstem synchronizing pathway in parallel with hippocampal theta-related cells. Hippocampus 5:306-319.

Dickson CT, Magistretti J, Shalinsky M, Hamam B, Alonso A (2000) Oscillatory activity in entorhinal neurons and circuits: Mechanisms and function. Ann NY Acad Sci 911:127-150.

Dickson CT, Trepel C, Bland BH (1994) Extrinsic modulation of theta field activity in the entorhinal cortex of the anesthetized rat. Hippocampus 4:37-52.

Dolorfo CL, Amaral DG (1998) Entorhinal cortex of the rat: organization of intrinsic connections. J Comp Neurol 398:49-82.

Eckhorn R, Bauer R, Jordan W, Brosch M, Kruse W, Munk M, Reitboek HJ (1988) Coherent oscillations: a mechanism of feature linking in the visual cortex? Biol Cybern 60:121-130.

Fellous J-M, Sejnowski TJ (2000) Cholinergic induction of oscillations in the hippocampal slice in the slow $(0.5-2 \mathrm{~Hz})$, theta $(5-12 \mathrm{~Hz})$, and gamma $(35-70 \mathrm{~Hz})$ bands. Hippocampus 10:187-197.

Fisahn A, Pike FG, Buhl EH, Paulsen O (1998) Cholinergic induction of network oscillations at $40 \mathrm{~Hz}$ in the hippocampus in vitro. Nature 394:186-189.

Fukuda TKT (2000) Gap junctions linking the dendritic network of GABAergic interneurons in the hippocampus. J Neurosci 20:1519-1528.

Galarreta M, Hestrin S (1999) A network of fast-spiking cells in the neocortex connected by electrical synapses. Nature 402:72-75.

Gibson JR, Beierlein M, Connors BW (1999) Two networks of electrically coupled inhibitory neurons in neocortex. Nature 402:75-79.

Gray CM (1994) Synchronous oscillations in neuronal systems: Mechanisms and functions. J Comput Neurosci 1:11-38.

Gray CM, Singer W (1989) Stimulus-specific neuronal oscillations in orientation columns of cat visual cortex. Proc Natl Acad Sci USA 86:1698-1702.

Gray CM, Konig P, Engel AK, Singer W (1989) Oscillatory responses in cat visual cortex exhibit inter-columnar synchronization which reflects global stimulus properties. Nature 338:334-337.

Herculano-Houzel S, Munk MHJ, Neuenschwander S, Singer W (1999) Precisely synchronized oscillatory firing patterns require electroencephalographic activation. J Neurosci 19:3992-4010.

Jeffery KJ, Donnett JG, O'Keefe J (1995) Medial septal control of thetacorrelated unit firing in the entorhinal cortex of awake rats. NeuroReport 6:2166-2170.

Jefferys J, Traub R, Whittington M (1996) Neuronal networks for induced "40 Hz" rhythms. Trends Neurosci 19:202-208.

Jones RSG (1994) Synaptic and intrinsic properties of neurons of origin of the perforant path in layer II of the rat entorhinal cortex in vitro. Hippocampus 4:335-353.

Jones RSG, Buhl EH (1993) Basket-like interneurons in layer II of the entorhinal cortex exhibit a powerful NMDA-mediated synaptic excitation. Neurosci Lett 149:35-39.

Klink R, Alonso A (1997a) Morphological characteristics of layer II projection neurons in the rat medial entorhinal cortex. Hippocampus 7:571-583.

Klink R, Alonso A (1997b) Muscarinic modulation of the oscillatory and repetitive firing properties of entorhinal cortex layer II neurons. J Neurophysiol 77:1813-1828.

Kohler C (1986) Intrinsic connections of the retrohippocampal region in the rat brain. II. The medial entorhinal area. J Comp Neurol 246:149-169.

Laurent G (1996) Dynamical representation of odors by oscillating and evolving neural assemblies. Trends Neurosci 19:489-496.

Leung LS (1998) Generation of theta and gamma rhythms in the hippocampus. Neurosci Biobehav Rev 22:275-290.

Librizzi L, de Curtis M (1999) Simultaneous recording of changes in $\mathrm{pH}$, $\mathrm{K}+$, and vascular tone during epileptiform activity in the olfactory-limbic cortex. Soc Neurosci Abstr 25:842.

Lisman JE, Idiart MAP (1995) Storage of $7 \pm 2$ short term memories in oscillatory subcycles. Science 267:1512-1515.

Llinás R, Ribary U (1993) Coherent 40-Hz oscillation characterizes dream state in humans. Proc Natl Acad Sci USA 90:2078-2081.

Ma M, Leung L-WS (1999) Medial septum mediates the increase in postictal behaviors and hippocampal gamma waves after an electrically induced seizure. Brain Res 833:51-57.

Maloney KJ, Cape EG, Gotman J, Jones BE (1996) High-frequency $\gamma$ electroencephalogram activity in association with sleep-wake states and spontaneous behaviors in the rat. Neuroscience 76:541-555.

Mann-Metzer P, Yarom Y (1999) Electrotonic coupling interacts with intrinsic properties to generate synchronized activity in cerebellar networks of inhibitory interneurons. J Neurosci 19:3298-3306.

Metherate R, Cox CL, Ashe JH (1992) Cellular bases of neocortical 
activation: modulation of neural oscillations by the nucleus basalis and endogenous acetylcholine. J Neurosci 12:4701-4711.

Mitchell SJ, Ranck JBJ (1980) Generation of theta rhythm in medial entorhinal cortex of freely moving rats. Brain Res 189:49-66.

Mitchell SJ, Rawlins JNP, Steward O, Olton DS (1982) Medial septal area lesions disrupt theta rhythm and cholinergic staining in medial entorhinal cortex and produce impaired radial arm maze behavior in rats. J Neurosci 2:292-302.

Muhlethaler M, de Curtis M, Walton K, Llinás R (1993) The isolated and perfused brain of the guinea pig in vitro. Eur J Neurosci 5:915-926.

Munk MHJ, Roelfsma PR, Konjig P, Engel AK, Singer W (1996) Role of reticular activation in the modulation of intracortical synchronisation. Science 227:271-274.

Murthy VN, Fetz EE (1992) Coherent 25 to $35 \mathrm{~Hz}$ oscillations in the sensorimotor cortex of awake behaving monkeys. Proc Natl Acad Sci USA 89:5670-5674.

Penttonen M, Kamondi A, Acsady L, Buzsaki G (1998) Gamma frequency oscillation in the hippocampus of the rat: intracellular analysis in vivo. Eur J Neurosci 10:718-728.

Ritz R, Sejnowski TJ (1997) Synchronous oscillatory activity in sensory systems: new vistas. Curr Opin Neurobiol 7:536-546.

Schurmann M, Basar-Eroglu C, Basar E (1997) Gamma responses in the EEG: elementary signals with multiple functional correlates. NeuroReport 8:1793-1796.

Shulz DE, Sosnik R, Ego V, Haidarliu S, Ahissar E (2000) A neuronal analogue of state-dependent learning. Nature 403:549-553.

Singer W, Gray CM (1995) Visual feature integration and the temporal correlation hypothesis. Annu Rev Neurosci 18:555-586.

Squire LR, Zola-Morgan S (1991) The medial temporal lobe memory system. Science 253:1380-1386.

Steriade M, Curró Dossi R, Paré D, Oakson G (1991) Fast oscillations $(20-40 \mathrm{~Hz})$ in thalamocortical systems and their potentiation by mesopontine cholinergic nuclei in the cat. Proc Natl Acad Sci USA 88:4396-4400.

Steward O, Scoville SA (1976) The cells of origin of entorhinal afferents to the hippocampus and fascia dentata of the rat. J Comp Neurol 169:347-370.

Tallon-Baudry C, Bertrand O (1999) Oscillatory gamma activity in humans and its role in object representation. Trends Cognit Sci 3:151-162.

Tamás G, Buhl EH, Lorincz A, Somogyi P (2000) Proximally targeted GABAergic synapses and gap junctions synchronize cortical interneurons. Nat Neurosci 3:366-371.

Tiitinen H, Sinkkonen J, Reinikainen K, Alho K, Lavikainen J, Naatanen R (1993) Selective attention enhances the auditory 40-Hz transient response in humans. Nature 364:59-60.

Traub R, Whittington M, Colling S, Buzsaki G, Jefferys J (1996) Analysis of gamma rhythms in the rat hippocampus in vitro and in vivo. J Physiol (Lond) 493:471-484.

van der Linden S, de Curtis M, Panzica F (1999) Carbachol induces fast oscillations in the medial but not in the later entorhinal cortex of the isolated guinea pig brain. J Neurophysiol 82:2441-2450.

Van Hoesen GW (1982) The parahippocampal gyrus. New observations regarding its cortical connections in the monkey. Trends Neurosci $5: 345-350$.

Wang X-J, Buzsaki G (1996) Gamma oscillation by synaptic inhibition in a hippocampal interneuronal network model. J Neurosci 16: $6402-6413$.

Whittington M, Traub RD, Jefferys JGR (1995) Syncronized oscillations in interneuron networks driven by metabotropic glutamate receptor activation. Nature 373:612-615.

Winson J (1978) Loss of hippocampal theta rhythm results in spatial memory deficit in the rat. Science 201:160-163.

Witter MP, Groenewegen HJ, Lopes da Silva FH, Lohman AHM (1989) Functional organization of the extrinsic and intrinsic circuitry of the parahippocampal region. Prog Neurobiol 33:161-253.

Wouterlood PG, Härtig W, Brückner G, Witter MP (1995) Parvalbuminimmunoreactive neurons in the entorhinal cortex of the rat: localization, morphology, connectivity and ultrastructure. J Neurocytol 24:135-153.

Zola-Morgan S, Squire LR (1993) Neuroanatomy of memory. Annu Rev Neurosci 16:547-563. 